\title{
ON SYSTEMATIC ERRORS IN THE MEASURED INTERVAL BETWEEN CLOSE LINES
}

In the year 1897, after a considerable number of Mills three-prism spectrograms had been measured, I noticed an interesting form of systematic error of measurement which led at once to the taking of precautions to eliminate its effects. The components of each of the two pairs of iron comparison lines at $4250 \mathrm{~A}$ and $4271 \mathrm{~A}$ are about two-thirds of an angstrom unit apart and clearly separated on the spectrograms, but the measures made the interval between the components always smaller than the wave-lengths determined by Rowland required. ${ }^{1}$ This was true in both systems of measurement: $(a)$ in which the micrometer wire was made to bisect each line, and $(b)$ in which the wire was set against each side of the line and the mean of the readings in the two positions taken as the reading on the center of the line; but the discrepancy (in my measures at least) was the greater by method (a). It seemed clear that the measure of one or both of the lines was affected by the presence of the close companion, in the sense that the interval between the centers of the lines was measured too small. It was sought to eliminate the effect upon the velocities of stars resulting from the individual absorption lines in the spectral regions concerned by using the mean of the readings on the two lines of each pair as the zero reading for the mean of the wave-lengths of the lines of the pair.

The next three observers had personal equations of the same sign as mine, and larger, but Dr. Burns's measures showed a small discrepancy of the opposite sign.

At my request Miss Hobe has copied from the records the measures of the two pairs of lines on some of the spectrograms secured from 1896 to 1902 with the original Mills spectrograph and has combined and tabulated them as below. There is no reason to expect

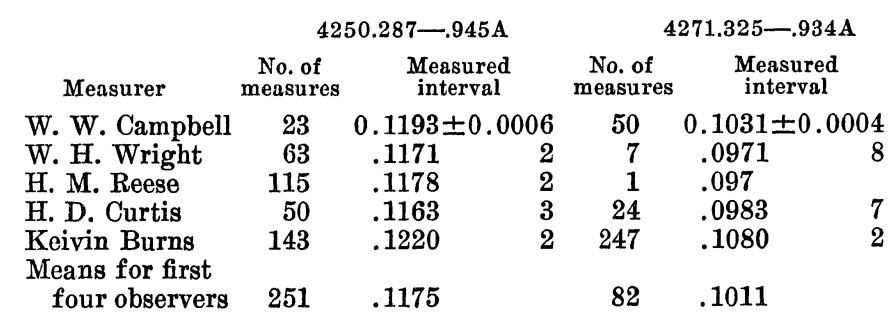

1 The wave-lengths which I used for these pairs of lines were those assigned by Rowland to the corresponding absorption lines in the solar spectrum; at that time we seemed not to know the wave-lengths in the laboratory spectrum of iron so accurately as we knew the wave-lengths of the iron lines in the solar spectrum. The discrepancies do not modify the conclusions of this paper appreciably. that the measures not used in making the tabulation would give different results. The value of one revolution of the micrometer serew is $r=0.5 \mathrm{~mm}$.

The computed intervals for Rowland's wavelengths and for Burns's ${ }^{2}$ wave-lengths, from the reduction curve which fits a long region of the Mills spectra, are:

$\begin{array}{lll} & 4250 \mathrm{~A} & 4271 \mathrm{~A} \\ \text { Rowland } & 0.1209 & 0.1082 \\ \text { Burns } & 0.1206 & 0.1054\end{array}$

Expressed in angstrom units, the intervals between the pairs of lines in Rowland's and Burns's tables are as in columns two and three below, and the intervals as measured by the first four observers and by Keivin Burns are as in columns four and five.

\begin{tabular}{lcllll} 
& \multicolumn{2}{c}{ Laboratory } & \multicolumn{2}{c}{ Mills spectrograph } \\
Pairs of lines & $\Delta$ Rowland & $\Delta$ Burns & 4 observers & K. Burns \\
4250.287-.945 & $0.658 \mathrm{~A}$ & $0.657 \mathrm{~A}$ & $\mathbf{0 . 6 3 9 A}$ & $0.664 \mathrm{~A}$ \\
4271.325-.934 & 0.609 & 0.593 & 0.569 & 0.608
\end{tabular}

We encountered several interesting examples of the phenomenon in October, 1915, when we attempted to construct a reduction curve for the region $4713 \mathrm{~A}$ to $5016 \mathrm{~A}$, from the laboratory spectra of iron, titanium, hydrogen and helium, to be used in determining the wave-lengths of the green nebular lines (Lick Obs. Bull., 9, 6, 1916). The high dispersion or interferometer positions of the laboratory lines that did not have companion lines nearer than two or three angstroms fell upon the curve, within the usual unavoidable errors; but we could not get close though clearly separated lines to fall on the curve. When the helium and iron spectra were photographed upon the same plate, for example, the helium line at $4922.1 \mathrm{~A}$ always fell above the curve of the iron lines by an amount three or four times the possible accidental error of observation. This discrepancy repeated itself on several plates. It was attributed to the influence of the strong iron line at $4920.8 \mathrm{~A}$ upon the micrometer measures. We then placed a diaphragm immediately in front of the photographic plate which cut out the iron lines that would have fallen within a few angstroms of the helium lines at $4922 \mathrm{~A}$ and 5015A. The iron spectrum was photographed with the diaphragm in place and the helium spectrum with the diaphragm removed. The helium lines were then found to lie upon the iron dispersion curve, and in the same manner upon the titanium curve.

2 Lick Obs. Bull., 8, 37, 1913. 
It seems clear, from these results, that observers may readily over-estimate the accuracy or reliability of measured intervals between pairs of bright lines that are close but well separated, and that different observers may have quite different systematic errors.

When I was measuring stellar spectrograms I received the impression that the same kind of error may occur in measuring the interval between close absorption lines, and that the observer's judgment in bisecting such lines is affected by the degree of darkening of the continuous spectrum. The mean of the measured positions of two such lines seems to be reliable, but the results for the individual lines may have quite small weight. If one line of such a pair is unsuitable for measurement, on account of unsymmetrical distribution of the silver grains or for other reasons, both lines of the pair should usually be rejected. The close pairs of stellar absorption lines chiefly concerned in forming the impression were those at $4250 \mathrm{~A}$ and $4271 \mathrm{~A}$; but as that region of the spectrum on the three-prism plates frequently suffered from different degrees of under-exposure, I did not pursue the subject to a definite conclusion.

There seems to be no reason why laboratory determinations of the wave-lengths of very close lines may not be affected with errors of the same kind.

April 5, 1916.
W. W. Campbell.

\title{
WOLF-RAYET BANDS IN THE NUCLEI OF THREE NEBULAE:
}

\author{
N. G. C. 6826, N. G. C. $418_{2}$, N. G. C. 40
}

Among the plates taken in connection with Dr. Campbell's programme for the determination of the radial velocities of the planetary nebulae a few have shown the spectrum of the nucleus. Since the nuclei of several planetary nebulae have recently been found to have the Wolf-Rayet character of spectrum, ${ }^{1}$ a description of the nuclear spectra shown on the above-mentioned plates will be of interest. With Dr. Campbell's permission the data furnished by the plates are here presented.

A bright band at about $4686 \mathrm{~A}$ in the spectrum of the nucleus of the planetary nebula N. G. C. 6826 was noticed by Dr. P. W. Merrill while measuring a oneprism plate taken August 9, 1913. This was recognized as the well-known Wolf-Rayet radiation at $4686 \mathrm{~A}$ common to both the Wolf-Rayet stars and the nebulae. Further examination discloses another less conspicuous bright band at about $4657 \mathrm{~A}$ which is not given in Dr. Campbell's list ${ }^{2}$ of Wolf-Rayet bands. Keeler $^{3}$ observed N. G. C. 6826 and glimpsed bright points in the continuous spectrum, but could not measure their positions. Measurement of the plate gives the following wave-lengths and approximate widths :

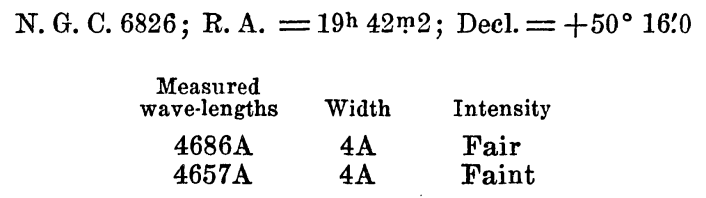

${ }^{1}$ W. H. Wright, Ap. Jour., 40, 466, 1914; Proc. N. A. S., 1, 266, 1915; paper presented to the A. A. A. S., Palo Alto, 1915.

$2 A$. and $A p$., 13, 468, 470, 1894.

3 Publ. Lick Obs., 3, 212, 1894.
A one-prism plate of the nebula N. G. C. $418_{2}$ taken October 16, 1913, shows two bright bands in the continuous spectrum of the nucleus. Measurement of this plate gives the following details:

$$
\begin{aligned}
& \text { N. G. C. } 418_{2}=\text { D. M. }-12^{\circ} 1172 ; \text { R. A. }=5^{\text {h }} 22 \mathrm{~m} 9 \text {; } \\
& \text { Decl. }=-12^{\circ} 46^{\prime} \\
& \begin{array}{ccc}
\begin{array}{c}
\text { Measured } \\
\text { wave-lengths }
\end{array} & \text { Width } & \text { Intensity } \\
4686 \mathrm{~A} & 3 \mathrm{~A} & \text { Faint } \\
4650 \mathrm{~A} & 8 \mathrm{~A} & \text { Fair }
\end{array}
\end{aligned}
$$

A conspicuous bright band was seen in the spectroscope upon observing the nebula N. G. C. 40, on October 6, 1913. The exposure then made was not sufficiently long to secure any of the nebular lines, and the plate shows the bright band alone. This band was at once measured and found to correspond to a wave-length of $4652 \mathrm{~A},{ }^{4}$ which is that of a well-known Wolf-Rayet band. This was, so far as known, the first instance of its observation in connection with a nebula. Another plate of N. G. C. 40 secured on September 26, 1914, with three prisms and 5-inch camera, was given longer exposure and careful guiding of the nucleus upon the mid-point of the slit. The slit was lengthened to admit the full diameter of the nebular image. As this plate contains a great deal of detail it has been thoroughly measured and will be described in connection with a brief description of the nebula itself. The results of the measurement are:

4 The wave-length of this band is given as $4688 \mathrm{~A}$ in the Harvard List of Gaseous Nebulae, Annals H. C. O., 56, 175, 1912 , and in the classification of the Spectra of Gaseous Nebulae, Annals H. C. O., 76, 21, 1915. This must have been a misidentification. 